\title{
Volvulus of splenic flexure: an autopsy case
}

\author{
Erkol $\mathrm{H}^{1}$, Erkol $\mathrm{Z}^{2}$, Aybasti $\mathrm{N}^{3}$, Inal $\mathrm{M}^{4}$ \\ Abant Izzet Baysal University, Medical Faculty, General Surgery Department, Golkoy, Bolu, Turkey. \\ hayrierkol@gmail.com
}

\begin{abstract}
Objective: The definition of volvulus is an axial twist of a portion of the gastrointestinal tract along its mesentery. The involved bowel is obstructed partially or completely with a variable degree of arterial and venous occlusion. The colon is the most common site for volvulus. The splenic flexure is the least common site of colonic volvulus. Splenic flexure volvulus (SFV) is a very rare cause of colonic obstruction, constituting $1-2 \%$ of colonic volvulus. Mortality rate of the SFV cases is low.

Case report: We experienced a SFV case who was a 20-year-old male soldier. The case had come to the state hospital with complaints of severe left abdominal and lumbar pain and a medical history of relapsing urinary infection and nephrolithiasis. The doctor had hospitalized him with the diagnosis of paralytic ileus caused by nephrolithiasis. He had died after 14 hours and 35 minutes from hospitalization. Autopsy findings showed out that the death cause was generalized peritonitis due to gangrenous SFV.

Conclusion: In this paper, we presented this case and discussed its properties in the light of the literature data (Fig. 2, Ref. 7). Full Text in PDF www.elis.sk.

Key words: volvulus, splenic flexure volvulus, gangrenous volvulus, mortality.
\end{abstract}

Splenic flexure volvulus (SFV) is very rare cause of colonic obstruction, constituting 1-2\% of colonic volvulus. Very few cases have been reported in the literature (1-3). Mortality rate of the SFV cases is low $(1,4)$. In this paper, a 20 -year-old male (soldier) case, who had died from generalized peritonitis caused by gangrenous SFV was presented and discussed with the literature data.

\section{Case report}

A 20-year-old male (soldier) had come to the infirmary at 9.00 AM o'clock, because of the pain at the left upper abdominal region. Relapsing urinary infection and nephrolithiasis had been determined at his medical history. He had also been cured for urinary infection in the infirmary before. Because the infirmary's doctor had suspected that the pain was caused by nephrolithiasis, he had injected him (IM) two analgesics and one spasmolytic drug during the eight hours. Then, the infirmary's doctor had brought him to the state hospital, because the pain was getting worse and he couldn't perform any more medical analysis in the infirmary's simple condition.

${ }^{1}$ Abant Izzet Baysal University, Medical Faculty, General Surgery Department, Golkoy, Bolu, Turkey, ${ }^{2}$ Abant Izzet Baysal University, Medical Faculty, Forensic Medicine Department, Golkoy Bolu, Turkey, ${ }^{3}$ Gaziantep University, Medical Faculty, General Surgery Department Gaziantep, Turkey, and ${ }^{4}$ Ceyhan State Hospital, General Surgery Clinic, Adana, Turkey

Address for correspondence: $\mathrm{H}$. Erkol, MD, Abant Izzet Baysal University, Izzet Baysal Medical Faculty, General Surgery Department, 14280 Golkoy, Bolu, Turkey.

Phone: +90.374.2534656, Fax: +90.374.2534615

Acknowledgement: This article was presented as a poster presentation in "IIIrd Congress of the Balkan Academy of Forensic Sciences", 2-5 June 2005, Constanta, Romania.
When the patient had come to the state hospital at 5.00 PM o'clock, he had severe left abdominal and lumbar pain. Air image, whose diameter was 20 centimeters, had been seen on the upper left region, at the abdominal direct graph. Aero - liquid line had also been seen on this graph. Because he couldn't urinate, urine sample could been taken by a urinary catheter and 7-8 leucocytes, 5-6 kidney epithelial cells, 7-8 calcium oxalate crystals and rare bacteria had been observed on this sample's analysis. The doctor had thought that nephrolithiasis couldn't be seen at the abdominal direct graph, taken in the emergency conditions, because of the air image. Therefore, after a nasogastric catheter had been fixed to the patient, IV serum infusion was applied to the patient for the diagnosis of "paralytic ileus caused by nephrolithiasis". Serum infusion had continued along the night. He had vomited once at the hospital. The patient had died at 7.35 AM o'clock, after 14 hours and 35 minutes from hospitalization.

It was learnt that the case had a complaint of chronic constipation. There wasn't any more information about examination findings in the hospital documents as well as in the other witness' expressions. Any more investigation hadn't been performed, like blood analysis, US, colon graph with contrast etc.

We performed the autopsy after 5.5 hours from death. Rigor mortis was maximally on all junctions and abdominal muscles. Abdominal region was seen extremely distended on the external examination.

Consequently, abdominal cavity was incised firstly. Dirty yellow - greenish colored, infectious, anaerobic smelling liquid discharged from abdominal cavity. Ascending colon and $2 / 3$ right side of transverse colon had become extremely wide, so their diameter was about 20 centimeters. The diameter of $1 / 3$ left side of the transverse colon was 30 centimeters. The wall had become thin and gangrenous (necrotic). Splenic flexure had returned 360 degrees to the 


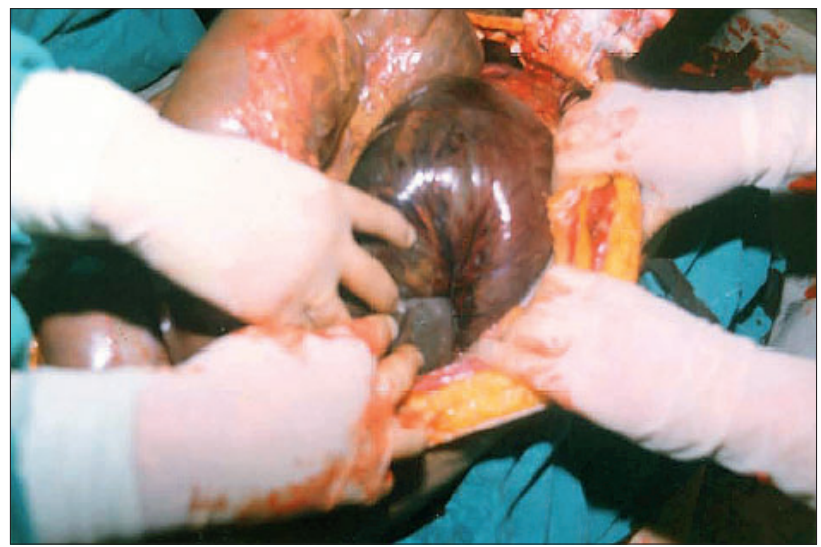

Fig. 1. Splenic flexure returned 360 degrees reversely to the course of watch dial; around its mesentery, then "closed loop obstruction" occurred.

reverse course of watch dial around its mesentery, then "closed loop obstruction" had occurred. Diameter of $1 / 2$ upper part of the descending colon was 30 centimeters and its wall was thin and necrotic too. There were some regions upon perforate on this wide colon segment. Diameters and appearances of ileum, jejunum and $1 / 2$ lower part of the descending colon were normal. Suppurative, anaerobic smelling liquid was seen around all internal organs and peritonitis was seen on the abdominal cavity examination (Figs 1 and 2).

When the tortioned splenic flexure returned 360 degrees to the course of watch dial around its mesentery; internal material of the colon, air and suppurative feces, passed into distal colon with a noise. There was no stone in the kidneys, ureters, urinary bladder and urethra. All the abdominal internal organs were intact and slightly autolytic. Edema in the cerebrum and the lungs, and subpleural petechial hemorrhages were observed.

At the end of the autopsy, it was determined that the death was due to generalized peritonitis from gangrenous SFV.

\section{Discussion}

The definition of volvulus is an axial twist of a portion of the gastrointestinal tract along its mesentery. The involved bowel is obstructed partially or completely with a variable degree of arterial and venous occlusion. The colon is the most common site for volvulus. The splenic flexure (SFV) is the least common site of colonic volvulus (4). Primary SFV is a rare entity (5). Primary SFV is due to congenital absence or laxity of the phrenocolic, gastro colic, and splenocolic ligaments while secondary volvulus is due to other causes including some prior surgery releasing these ligaments. A preoperative diagnosis can be established based on the characteristic radiological findings on plain x-ray abdomen and CT scan (2). Mittal et al (2007) presented a primary SFV case with gangrene in a 24-year-old man. They mentioned that radiograph had showed a massively dilated large bowel loop with a coffeebean sign and at emergency laparotomy, a distended and gangrenous splenic flexure had been found, with absence of all three ligamentous attachments of the splenic flexure (5).

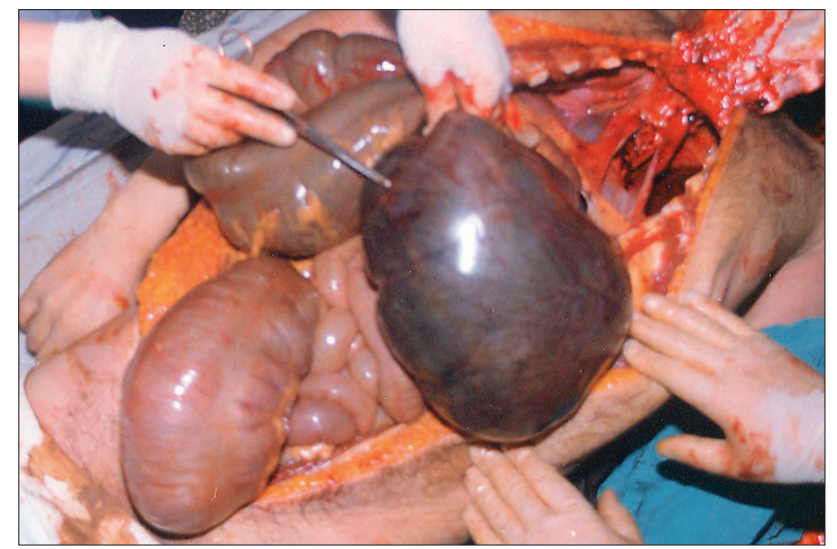

Fig. 2. Tortioned splenic flexure wall was thin and necrotic. There were some regions upon perforate on this wide colon segment.

Ballantyne (1981) reported the first case of gangrenous colon from volvulus of the splenic flexure. He had reviewed 14 detailed case reports of splenic flexure volvulus in the literature and mentioned that patients were in average 53.2 years old. Eight of fourteen were women. Previous abdominal surgery, anomalies of fixation, and constipation played important roles in the pathogenesis. Diagnosis had been made before surgery in two-thirds of the patients. Eleven had required emergency surgery. Three underwent operative detorsion, six partial colectomy. He had applied exteriorization of the splenic flexure as a loop colostomy on the case presented by him. He told that, there was only one death in fourteen cases, a $7 \%$ mortality rate and this low mortality rate was attributed to the rarity of gangrenous colon from SFV (1).

Machado et al (2009) presented a case of SFV in a young man who had acute abdominal pain, and distension, and illustrated the usefulness of CT scan, and plain x-ray of the abdomen in making a preoperative diagnosis. They told that, laparotomy had revealed a gangrenous SFV, which had been resected and primary anastomosis had been carried out (2).

Rath et al (2003) reported a case of a young female who had primary SFV due to absence of ligamental attachment. They told that a resection of the volvulus segment with end to end anastomosis was the curative surgical treatment (3).

Cho et al (1994) presented a case of the SFV. They told that there were 29 SFV cases in English literature and their woman case was 30th of them. They mentioned that, the case, a 30-year-old woman, had come to hospital with abdominal pain, distention and vomiting. There had been "bird beak" view on the colon graph with contrast. Colon and splenic flexure resection had been performed in the operation, then the operation results were excellent (4).

Majahan et al (2000) reported a SFV case in a patient who had previously been operated upon for a sigmoid volvulus (6).

Zago et al (1992) presented a case of perforated SFV treated with resection, end colostomy and closure of the distal stump. They mentioned that their case was 30th SFV case and 6th case with ischemic complications and the only one with perforation recorded till publication date in the literature (7). 
508-510

In our case, the initial symptom was pain at the left upper abdominal region. Infirmary's doctor had injected him two analgesic and one spasmolytic agent IM because he had thought that the history of relapsing urinary infection had originated from nephrolithiasis. He couldn't perform any analysis in the infirmary's simple condition.

Although the doctor of state hospital had known that these injected agents could masked acute abdomen symptoms (rebound, defence, etc) and the patient couldn't give the urine sample for analysis, probably due to septic shock, the doctor had thought that the cause of this pain was paralytic ileus caused by nephrolithiasis. There were no more physical examination findings of the case until his death in the hospital document. Since the findings of physical examination in medical document were so insufficient and more medical investigation (blood analysis, US, CT, colon graph with contrast etc.) hadn't been performed, this SFV case's, which is seen so rare in literature, specific proofs couldn't be evaluated and discussed. Any underlying factor for SFV, wasn't found at the autopsy, like absence of colonic bindings, previous operation history etc. Only, it was learnt that the case had a complaint of chronic constipation. As the Ballantyne mentioned, SFV's low mortality rate was attributed to the rarity of gangrenous colon from SFV. Therefore, since our case was a gangrenous SFV, this situation could be evaluated as a risk factor that increases mortality. On the other hand, Attorney Generalship began an investigation because of the complaints of the deceased's family, who claim that the state hospital's doctor had showed neglect and deficiency during the observation and therapy of this patient.

Although SFV cases are so rare, it must be considered at the distinctive diagnosis because lethal outcome cases are rarer. In this way, these kinds of cases may be diagnosed and treated successfully.

\section{References}

1. Ballantyne GH. Volvulus of the splenic flexure: report of a case and review of the literature. Dis Colon Rectum 1981; 24 (8): 630-632.

2. Machado NO, Chopra PJ, Subramanian SK. Splenic flexure volvulus presenting with gangrene. Saudi Med J 2009; 30 (5): 708-711.

3. Rath PK, Abbas M, Mishra B, Narayan. Primary splenic flexure volvulus--a case report with review of literature. J Indian Med Assoc 2003; 101 (2): 110-112.

4. Cho YU, Sohn SK, Chi HS, Kim KW. Volvulus of the splenic flexure of the colon. Yonsei Med J 1994; 35 (1): 97-100.

5. Mittal R, Samarasam I, Chandran S, Mathew G. Primary splenic flexure volvulus. Singapore Med J 2007; 48 (3): e87-89.

6. Mahajan R, Seth S, Braithwaite PA. Volvulus of the splenic flexure of colon: a case report and review. Int J Colorectal Dis 2000; 15 (3): 182-184.

7. Zago A, Pollini GP, Gandolfi P, Moretto G, Ferrara R, Donati CL, Mazzilli G. Rare colonic occlusions: volvulus of the splenic flexure. Ann Ital Chir 1992; 63 (4): 501-503. 